\title{
Simultaneous Quantitative Determination of Monosaccharides Including Fructose in Hydrolysates of Yogurt and Orange Juice Products by Derivatization of Monosaccharides with $p$-Aminobenzoic Acid Ethyl Ester Followed by HPLC
}

\author{
Joung Ho Ko, Huazi Huang, Gyoung Won Kang, and Won Jo Cheong* \\ Department of Chemistry and Institute of Basic Research, Inha University, Incheon 402-751, Korea \\ *E-mail: wjcheong@inha.ac.kr \\ Received May 3, 2005
}

\begin{abstract}
We have determined the contents of monosaccharides in the hydrolysates of some yogurt and orange juice products by derivatizing monosaccharides with $p$-aminobenzoic acid ethyl ester (ABEE). The separation of the ABEE-derivatized monosaccharides was efficiently carried out by HPLC using a microcolumn packed with the Alltima $\mathrm{C}_{18}$ stationary phase. The concentrations of monosaccharides were determined based on the measured peak area/height counts. ABEE derivatization of fructose and its detection have never been successfully carried out before this work. In this study, two peaks were observed in a fixed ratio for ABEE-fructose, and the ratio was maintained over a wide range of fructose concentration. In order to prove the validity of the above method, we compared the concentrations of glucose, galactose and fructose determined by ABEE derivatization and UVD (ultraviolet detector) chromatography with those determined by RID (refractive index detector) chromatography without derivatization. The determined concentrations of monosaccharides obtained from the two chromatographic methods were found close to each other within acceptable error ranges.
\end{abstract}

Key Words : Monosaccharides, Reductive amination, $p$-Aminobenzoic acid ethyl ester, HPLC

\section{Introduction}

Carbohydrates are among the most abundant compounds found in Nature, ${ }^{1}$ and the analysis of sugars and sugar mixtures is of considerable importance to food and beverage industry. The study on both qualitative and quantitative analyses of saccharides is a very complex and challenging area. Analysis of polysaccharides and oligosaccharides in lactic ferments demands even conformational and structural analysis. The first step of such complicated analysis of saccharides is determination of composition of monosaccharides. To determine the monosaccharides composition of a saccharide sample, it is usually hydrolyzed completely, derivatized to promote volatility (methylation or trimethylsilylation), and analyzed by GC/MS. The ion exchange chromatography system with a pulsed amperometric detector is also prevalent in determination of saccharides for its excellent performance. Quantitative determination of saccharides by a HPLC method with a refractive index detector has been hardly reported. Low sensitivity and inapplicability to gradient elution of refractive index detector retarded such approaches.

Carbohydrates, in general, do not absorb UV light. Thus chemical attachment of a chromophore to the carbohydrate molecule is often required to provide UV absorptivity at selected wavelengths. Derivatization methods enable the chromatographic analysis to achieve a sufficiently high sensitivity in the very low concentration range. ${ }^{2}$ A large number of derivatization reagents for monosaccharides have been reported in the literature, such as 3-methyl-1-phenyl-2pyrazolin, ${ }^{3,4} 8$-aminopyrene $1,3,6$-trisulfonate, ${ }^{5}$ benzamidine, ${ }^{6}$
FMOC-hydrazine,${ }^{7}$ phenylisocyanate,${ }^{8}$ 2-aminobenzoic acid,${ }^{9}$ $p$-aminobenzoic acid ethyl ester (ABEE) ${ }^{10-13}$ aminopyrazine. ${ }^{14}$ Among those, the interest of derivatization with $\mathrm{ABEE}$ has been recently increased. ABEE-derivatization of fructose and its determination, however, have not been reported in the literature so far. We successfully determined saccharides in commercial yogurt products by classes based on molecular weights by using a chromatography system with a $\mathrm{NH}_{2}$ column and a RID in the previous study. ${ }^{15}$ In this study, we have determined the compositions of monosaccharides in the hydrolysates of yogurt and orange juice products by two chromatography systems with a UVD and with a RID. In the chromatography system with a UVD, monosaccharides were derivatized with ABEE and separated in the Alltima $C_{18}$ column under optimized chromatographic conditions. Especially, separation and determination of ABEE-derivatized fructose was reported in this study for the first time. In the chromatography system with a refractive index detector, monosaccharides were separated in the Supelcogel $\mathrm{Pb}$ column with $100 \%$ water as the eluent. The determined results were in good agreement between the two chromatographic systems.

\section{Experimental Section}

Chemicals. Tetrahyrofuran and water were of HPLC grade and purchased from Fisher (Pittsburgh, PA, USA) and used without purification. Fructose, glucose, galactose, ABEE, sodium cyanoborohydride, sodium citrate, citric acid, and acetic acid were obtained from Sigma (St. Louis, MO, USA). 
Chromatography system. The chromatography system we used for ABEE-derivatized monosaccharides, was composed of a Shimadzu (Tokyo, Japan) LC-10AD pump, a Rheodyne (Cotati, USA) sample injection valve with $0.2 \mu \mathrm{L}$ loop, a Samsung (Seoul, Korea) SLC-200 UV detector, an Alltima $\mathrm{C}_{18}(0.5 \mathrm{~mm}$ I.D. $\times 300 \mathrm{~mm}$ length, $5 \mu \mathrm{m}$ particle size) column, and a computer equipped with the YoulinGisul (Sung Nam, Korea) Multichro 2000 software. The eluent was $88: 12$ mixture by volume of $100 \mathrm{mM}$ sodium citrate ( $\mathrm{pH} 5.5)$ and tetrahydrofuran. The flow rate was fixed at $0.01 \mathrm{~mL} / \mathrm{min}$.

A Supelco (Bellefonte, PA, USA) Supelcogel $\mathrm{Pb}$ column (78 mm I.D. $\times 300 \mathrm{~mm}$ length), a Shimadzu RID-6A refractive index detector, and a Rheodyne injector with 20 $\mu \mathrm{L}$ loop were used in the chromatography system for analysis of underivatized monosaccharides. Separation was carried out in $100 \%$ water at a flow rate of $0.5 \mathrm{~mL} / \mathrm{min}$ at 85 ${ }^{\circ} \mathrm{C}$.

Preparation of the hydrolyzed orange juice sample. Orange juice (Sunkist, Haitai) was filtered with a $0.45 \mu \mathrm{m}$ membrane filter. The filtered solution was hydrolyzed with $1.0 \mathrm{M} \mathrm{H}_{2} \mathrm{SO}_{4}$ at $85{ }^{\circ} \mathrm{C}$ for 6 hours, and neutralized with 0.5 $\mathrm{MNaOH}$.

Preparation of the hydrolyzed yogurt sample. Yogurt (Probio GG, Maeil) was centrifuged at $20,000 \mathrm{rpm}$ for 30 minutes and filtered with a $0.45 \mu \mathrm{m}$ membrane filter to remove solid particles. The filtered solution was hydrolyzed with $1.0 \mathrm{M} \mathrm{H}_{2} \mathrm{SO}_{4}$ at $85^{\circ} \mathrm{C}$ for 6 hours, and neutralized with $0.5 \mathrm{M} \mathrm{NaOH}$. The initial volume and final volume of the sample were measured.

Derivatization procedure to obtain ABEE-monosaccharides. An aliquot of $100 \mu \mathrm{L}$ hydrolyzed sample (or a standard solution) was mixed with $80 \mu \mathrm{L}$ of acetic acid, 80 $\mu \mathrm{L}$ of $1.4 \mathrm{M} \mathrm{NaBH}{ }_{3} \mathrm{CN}$, and $400 \mu \mathrm{L}$ of $\mathrm{ABEE}$, and the mixture was heated at $80{ }^{\circ} \mathrm{C}$ for $30 \mathrm{~min}$, and cooled to room temperature.

SPE (solid phase extraction). The hydrolyzed and neutralized sample contains salts that cause chromatographic interferences. We used the Sep-Pak $\mathrm{NH}_{2}$ (12 cc) cartridge to remove most of the interfering salts. First, we flushed the stationary phase with water and acetonitrile to activate it, then loaded an $1.0 \mathrm{~mL}$ aliquot of the hydrolyzed and neutralized yogurt sample. The cartridge was eluted with $3.0 \mathrm{~mL} 80 / 20(\mathrm{v} / \mathrm{v}) \% \mathrm{MeCN} /$ water to wash out salts. Then $100 \%$ water $(6 \mathrm{~mL})$ was used to recover monosaccharides, and recovered monosaccharides were collected in a bottle, evaporated to dryness, and dissolved in water for derivatization with $\mathrm{ABEE}$.

Recovery test. Two batches (1 mL each) of standard solutions containing $10.0 \mathrm{mg} / \mathrm{mL}$ of glucose, galactose, fructose, respectively, were prepared, to examine the recovery yield of the above SPE process. One batch was applied to the SPE process, and the recovered monosaccharides were dissolved in $1 \mathrm{~mL}$ water, and its chromatogram was obtained by the chromatography system with the RID and Supelcogel Pb column. The other batch was directly injected for chromatographic analysis. The measured peak counts of the two batches were used to calculate the recovery of each monosaccharide.

Quantitative determination of monosaccharides by UVD-HPLC. A standard sample of monosaccharides and a hydrolyzed sample were derivatized at the same time under the same condition and the measured peak counts of both samples were used for calculation of the concentration of each monosaccharide in the hydrolyzed sample. The crude concentration of each monosaccharide was determined in a preliminary experiment with a standard of arbitrary concentrations. Then a new standard was made with the determined concentrations of monosaccharides and this new standard and the hydrolyzed sample were now simultaneously derivatized for precise determination. This process was repeated three times. The final monosaccharide concentration in the original yogurt (or orange juice) solution was obtained by multiplying the determined concentration of the hydrolyzed sample with the reversal of the dilution factor measured during the experiment.

\section{Results and Discussion}

Recovery of SPE. The SPE recovery yields for glucose, galactose, and fructose were found to be $98.7 \pm 1.1 \%, 95.2 \pm$ $1.8 \%, 97.6 \pm 1.2 \%$, respectively.

Determination of monosaccharides in the hydrolyzed yogurt. Some commercial yogurt products were filtered and analyzed by the chromatography system with a RID, and the GG brand was chosen for this study since it had minimized impurities that cause problems in the derivatization and separation steps. The RID chromatogram of filtered GG yogurt is shown in the Figure 1. The Supelcogel column bears size exclusion characteristics: thus, monosaccharides eluted later than oligosaccharides and polysaccharides. As shown in Figure 1, the filtered GG yogurt contained lots of monosaccharides and some oligosaccharides.

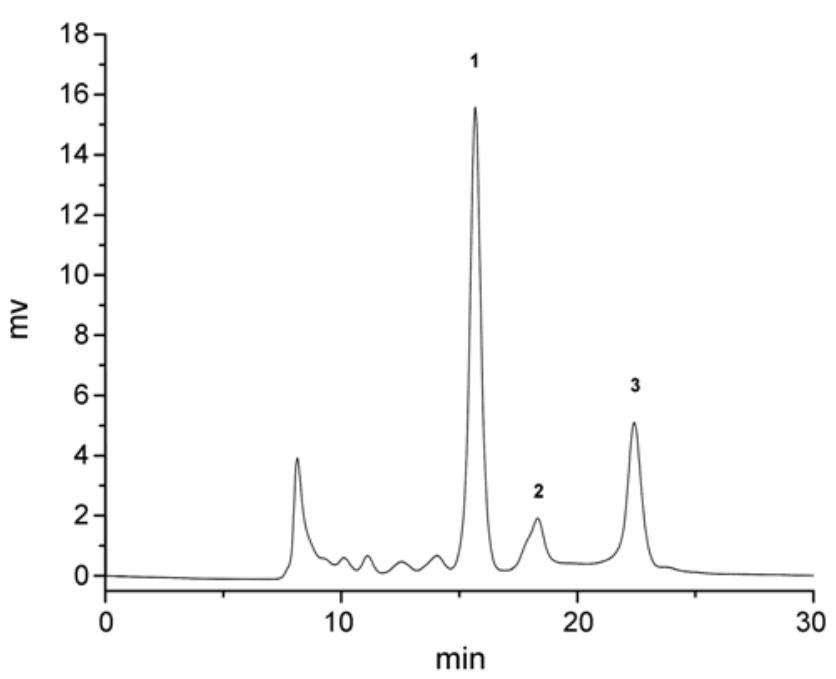

Figure 1. The RID chromatogram of the filtered Probio GG yogurt. The eluent was $100 \%$ water with the Supelcogel $\mathrm{Pb}$ column $(7.8$ mm I.D. $\times 300 \mathrm{~mm}$ ) and the flow rate was $0.5 \mathrm{~mL} / \mathrm{min}$ at $85^{\circ} \mathrm{C} .1$; glucose 2; galactose 3 ; fructose. 


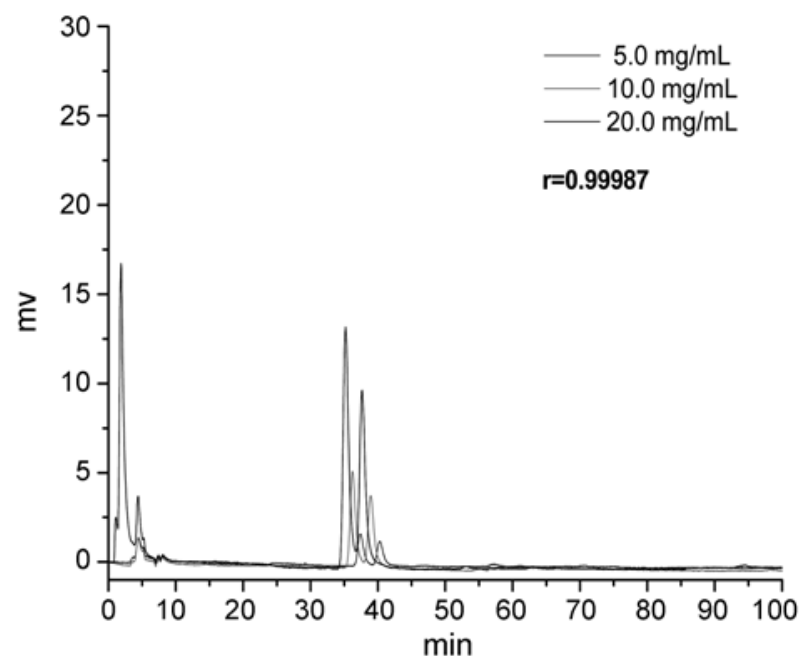

Figure 2. The chromatograms of the ABEE-fructose derivative at different concentrations. The eluent was $88: 12$ mixture by volume of $100 \mathrm{mM}$ sodium citrate $(\mathrm{pH} 5.5)$ and tetrahydrofuran for the Alltima $\mathrm{C}_{18}$ column $(0.5 \mathrm{~mm}$ I.D $\times 300 \mathrm{~mm}, 5 \mu \mathrm{m})$. The flow rate was $0.01 \mathrm{~mL} / \mathrm{min}$.
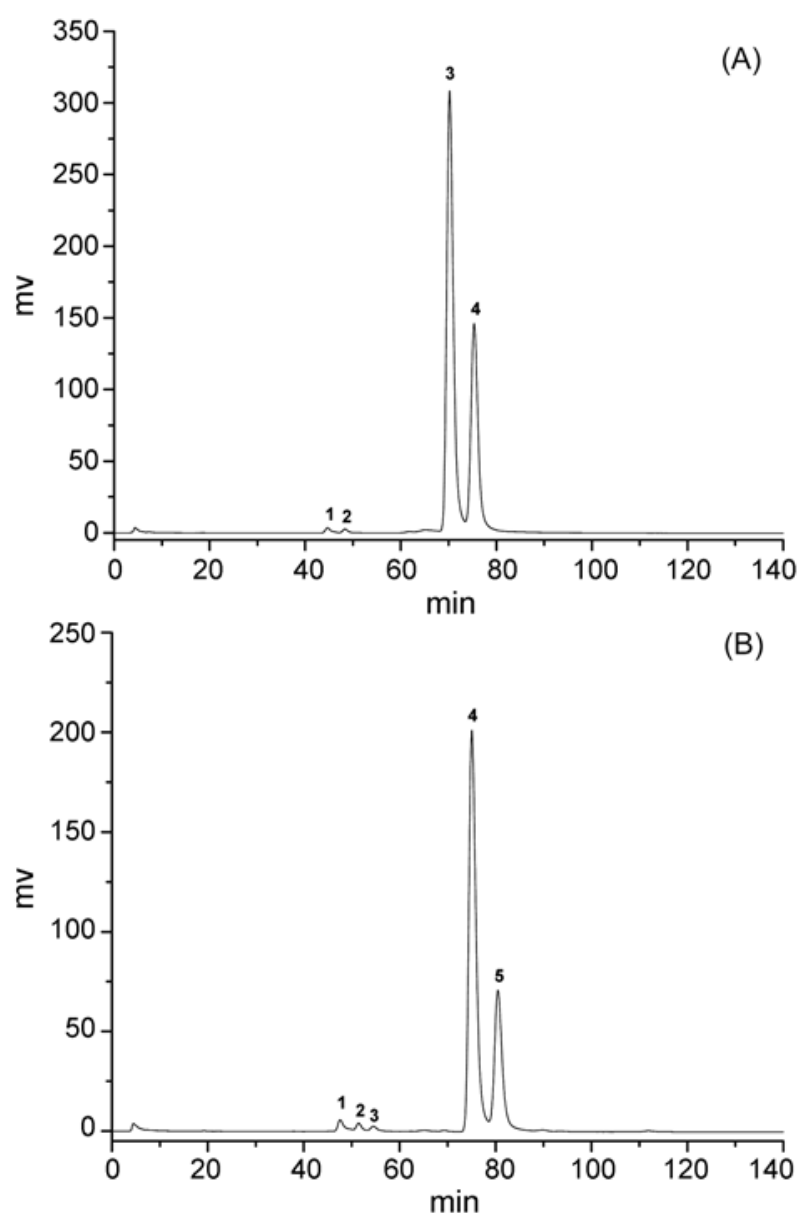

Figure 3. (A) The chromatogram of ABEE-derivatized monosaccharides of a standard. (B) The chromatogram of ABEE-derivatized monosaccharides of the hydrolyzed Probio GG yogurt. The eluent was $88: 12$ mixture by volume of $100 \mathrm{mM}$ sodium citrate $(\mathrm{pH} 5.5)$ and tetrahydrofuran for the Alltima $\mathrm{C}_{18}$ column $(0.5 \mathrm{~mm}$ I.D $\times 300 \mathrm{~mm}, 5 \mu \mathrm{m}$ ). The flow rate was $0.01 \mathrm{~mL} / \mathrm{min}$. (A) 1,2 ; fructose 3 ; glucose 4 ; galactose (B) 1,2 ; fructose 3 ; unknown 4 ; glucose 5; galactose.
ABEE-derivatized monosaccharides were nicely separated under the optimized separation conditions with the Alltima $\mathrm{C}_{18}$ stationary phase. Two peaks were observed for ABEEfructose while one peak was observed for any other ABEEderivatized monosaccharide. As mentioned before, ABEEderivatization of fructose and its detection have not been reported in the literature. Actually reductive amination of aldoses with ABEE is well described in the literature, but the same reaction of ketoses (like fructose) has not been mentioned so far, which seems to allude that reductive amination with $\mathrm{ABEE}$ is valid only for aldoses.

We found that, however, the ABEE-fructose could be formed and detected although the sensitivity in absorbance measurement of ABEEE-fructose was much lower than that of ABEE-aldose. The reason why ABEE-fructose shows such a low sensitivity and doublet peaks is not clear. We only guess that it may be related to the different structure of fructose from aldoses. Nevertheless, the peak signal of ABEE-fructose was linear with its concentration (Figure 2). The peak ratio of the doublet was also kept constant over a wide range of concentration. The chromatogram of ABEEderivatized monosaccharides for a standard and the hydrolyzed yogurt are compared in Figure 3. The chromatogram of ABEE-derivatized monosaccharides of the hydrolyzed Probio GG spiked with ABEE-fructose (Figure 4) shows that the peaks 1 and 2 are related to ABEE-fructose and that the peak 3 is an unidentified impurity.

The hydrolyzed yogurt sample was also analyzed by direct injection to the RID chromatography system with the Supelcogel $\mathrm{Pb}$ column to comparatively prove the validity of the above analytical approach, that is, derivatization followed by detection with UVD-HPLC. The RID chromatograms are shown in Figure 5. The determined concentrations of monosaccharides based on peak area and height counts by

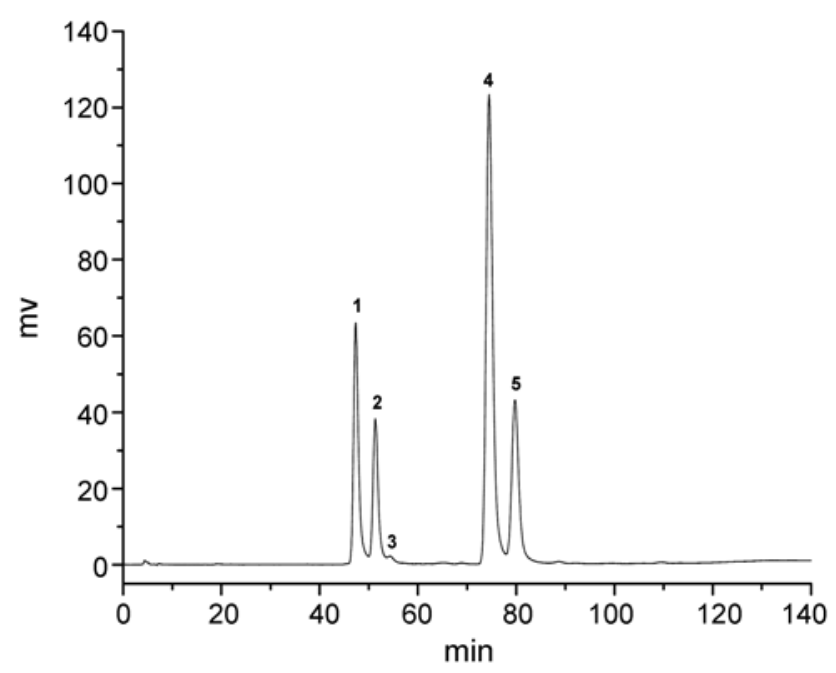

Figure 4. The chromatogram of ABEE-derivatized monosaccharides of the hydrolyzed Probio GG spiked with ABEE-fructose. The eluent was $88: 12$ mixture by volume of $100 \mathrm{mM}$ sodium citrate $\left(\mathrm{pH} \mathrm{5.5)}\right.$ and tetrahydrofuran for the Alltima $\mathrm{C}_{18}$ column $(0.5 \mathrm{~mm}$ I.D $\times 300 \mathrm{~mm}, 5 \mu \mathrm{m})$. The flow rate was $0.01 \mathrm{~mL} / \mathrm{min}$. 1,2 ; fructose 3 ; unknown 4; glucose 5; galactose. 

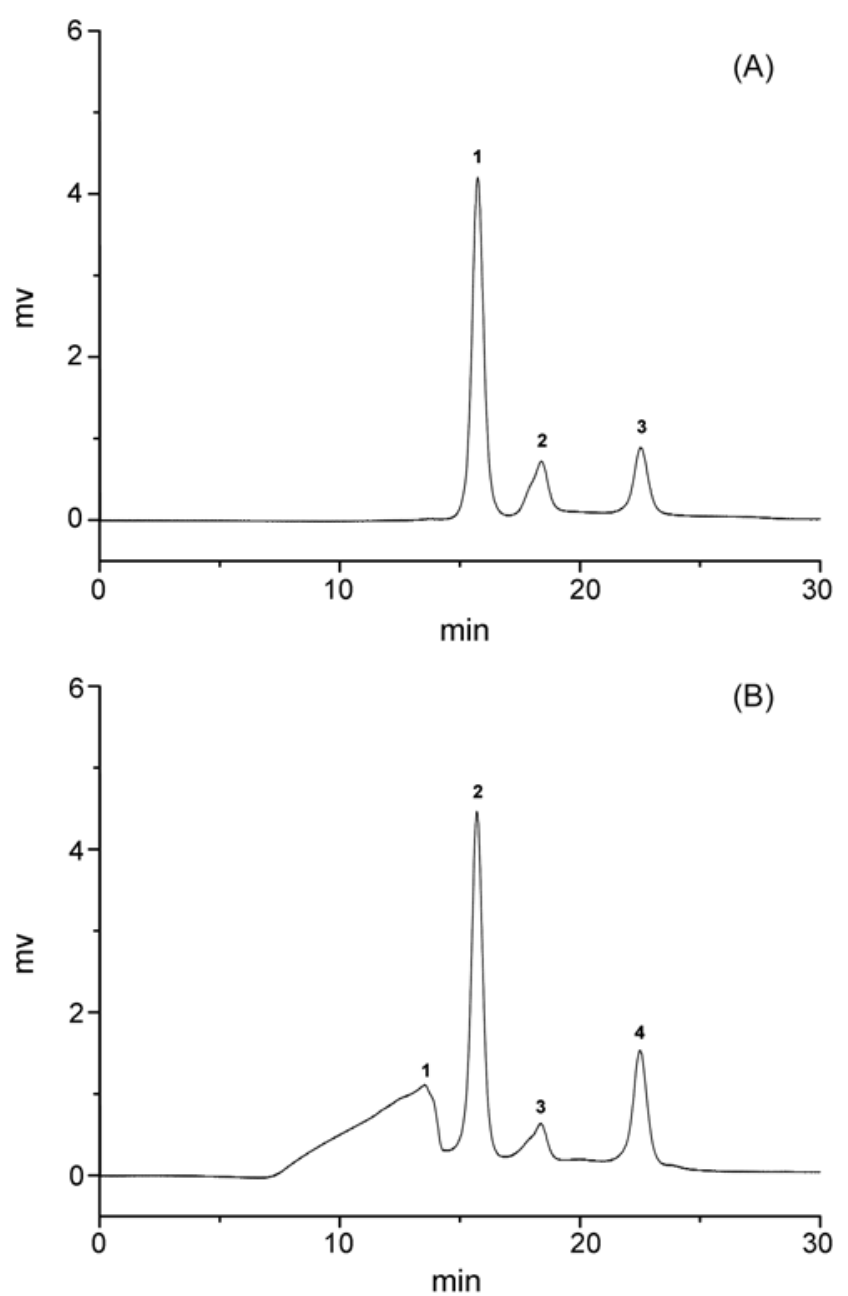

Figure 5. (A) The RID chromatogram of a standard. (B) The RID chromatogram of the hydrolyzed Probio GG yogurt. The eluent was $100 \%$ water with the Supelcogel $\mathrm{Pb}$ column (7.8 mm I.D. $\times$ $300 \mathrm{~mm}$ ) and the flow rate was $0.5 \mathrm{~mL} / \mathrm{min}$ at $85^{\circ} \mathrm{C}$. (A) 1 ; glucose 2; galactose 3; fructose (B) $1 ; \mathrm{Na}_{2} \mathrm{SO}_{4}$ (residual) 2; glucose 3; galactose 4; fructose.

both of the UVD-HPLC and RID-HPLC systems are comparatively summarized in Table 1 . Three different batches of the yogurt sample (same bottle) were analyzed on different days by each method. Reproducibilities were quite good for both of the UVD-HPLC and RID-HPLC systems. The measured concentrations were in good agreement between the two analytical approaches.

Determination of monosaccharides in the hydrolyzed orange juice. First, the orange juice sample was directly analyzed by the RID chromatography after filtration, and its chromatogram is shown in Figure 6. There were little oligosaccharides in the orange juice. Sucrose, glucose, and fructose were affluently identified instead. The UVD chromatograms of ABEE-derivatized monosaccharides for a standard and the hydrolyzed orange juice are given in Figure 7. The RID chromatograms of a standard and the hydrolyzed orange juice are given in Figure 8. The determined concentrations of monosaccharides by both of the UVD-HPLC and RID-HPLC systems are comparatively summarized in Table
Table 1. The determined concentrations of glucose, galactose, and fructose in the hydrolyzed GG yogurt sample by the UVD and RID chromatography systems

\begin{tabular}{|c|c|c|c|c|c|c|}
\hline \multicolumn{7}{|c|}{ UVD/HPLC SYSTEM } \\
\hline \multicolumn{7}{|c|}{ Concentration of monosaccharides based on peak area $(\mathrm{mg} / \mathrm{mL})$} \\
\hline & 1 & 2 & 3 & Average & SD & $\%$ RSD \\
\hline Glucose & 77.2 & 77.9 & 76.5 & 77.2 & 0.7 & 0.9 \\
\hline Galactose & 22.5 & 23.3 & 23.8 & 23.2 & 0.7 & 2.9 \\
\hline Fructose & 71.8 & 69.7 & 71.3 & 70.9 & 1.1 & 1.6 \\
\hline \multicolumn{7}{|c|}{ Concentration of monosaccharides based on peak heigth $(\mathrm{mg} / \mathrm{mL})$} \\
\hline Glucose & 77.8 & 76.4 & 77.0 & 77.1 & 0.7 & 0.9 \\
\hline Galactose & 22.9 & 23.3 & 24.2 & 23.5 & 0.6 & 2.7 \\
\hline Fructose & 69.0 & 69.9 & 69.5 & 69.5 & 0.4 & 0.6 \\
\hline
\end{tabular}

\section{RID/HPLC SYSTEM}

\begin{tabular}{l|c|c|c|c|c|c}
\hline \multicolumn{6}{c}{ Concentration of monosaccharides based on peak area $(\mathrm{mg} / \mathrm{mL})$} \\
\hline & 1 & 2 & 3 & Average & SD & $\%$ RSD \\
\hline Glucose & 78.8 & 79.2 & 79.8 & 79.3 & 0.5 & 0.6 \\
\hline Galactose & 23.9 & 23.5 & 23.4 & 23.6 & 0.3 & 1.2 \\
\hline Fructose & 71.8 & 71.8 & 70.9 & 71.5 & 0.5 & 0.7 \\
\hline
\end{tabular}

Concentration of monosaccharides based on peak height $(\mathrm{mg} / \mathrm{mL})$

\begin{tabular}{l|l|l|l|l|l|l}
\hline Glucose & 80.7 & 80.2 & 80.5 & 80.5 & 0.2 & 0.3 \\
\hline Galactose & 24.4 & 24.1 & 24.1 & 24.2 & 0.2 & 0.8 \\
\hline Fructose & 71.5 & 71.4 & 71.9 & 71.6 & 0.3 & 0.4 \\
\hline
\end{tabular}

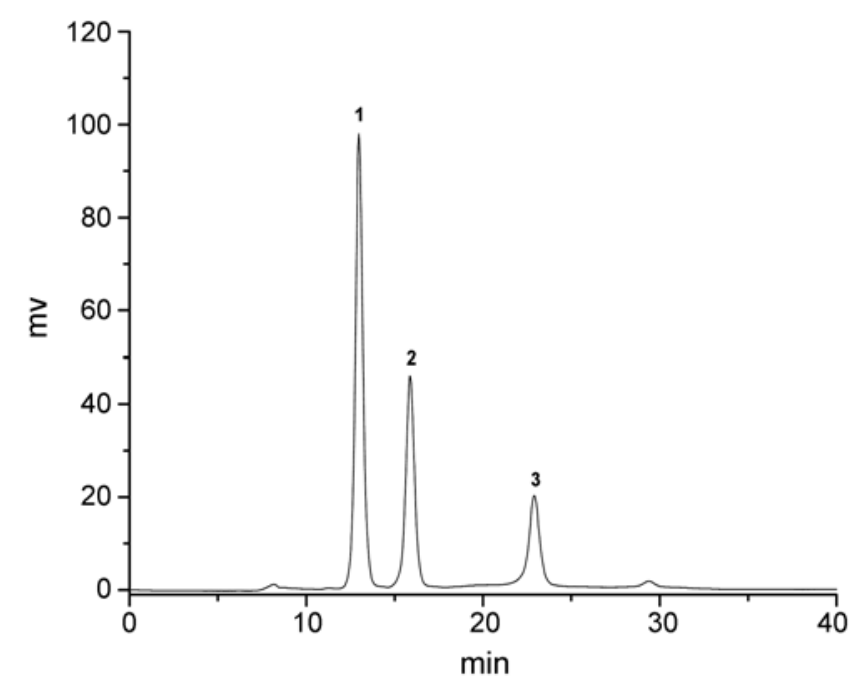

Figure 6. The RID chromatogram of the orange juice sample. The eluent was $100 \%$ water with the Supelcogel $\mathrm{Pb}$ column $(7.8 \mathrm{~mm}$ I.D. $\times 300 \mathrm{~mm})$ and the flow rate was $0.5 \mathrm{~mL} / \mathrm{min}$ at $85^{\circ} \mathrm{C}$. 1 ; sucrose 2; glucose 3; fructose.

2. Three different batches of the orange juice sample (same bottle) were analyzed on different days by each method. We can note again that reproducibilities of repeated analyses were quite good for both methods and that measured 

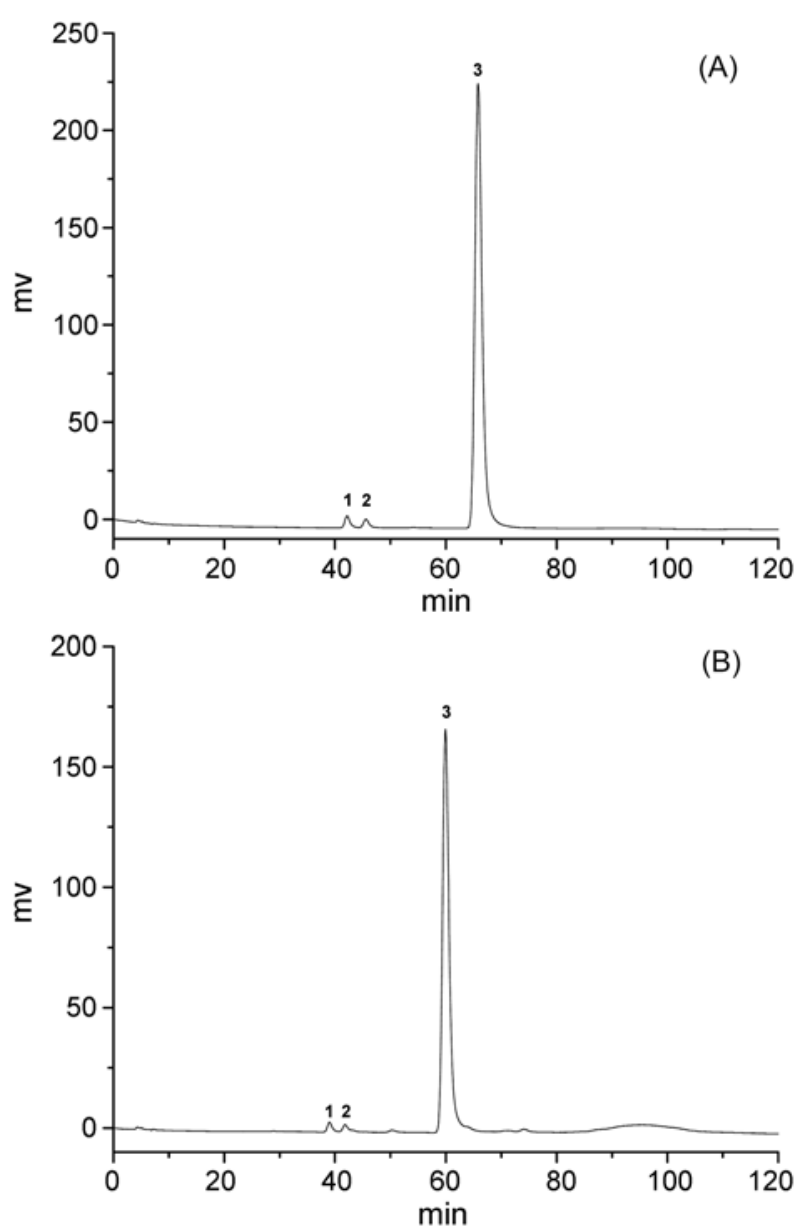

Figure 7. (A) The chromatogram of ABEE-derivatized monosaccharides of a standard. (B) The chromatogram of ABEE-derivatized monosaccharides of the hydrolyzed orange juice. The eluent was $88: 12$ mixture by volume of $100 \mathrm{mM}$ sodium citrate $(\mathrm{pH} 5.5)$ and tetrahydrofuran for the Alltima $\mathrm{C}_{18}$ column $(0.5 \mathrm{~mm}$ I.D $\times 300$ $\mathrm{mm}, 5 \mu \mathrm{m})$. The flow rate was $0.01 \mathrm{~mL} / \mathrm{min}$. 1,2; fructose 3; glucose.

Table 2. The determined concentrations of glucose and fructose in the hydrolyzed orange juice sample by the UVD and RID chromatography systems

\section{UVD/HPLC SYSTEM}

Concentration of monosaccharides based on peak area $(\mathrm{mg} / \mathrm{mL})$

\begin{tabular}{l|c|c|c|c|c|c}
\hline & 1 & 2 & 3 & Average & SD & $\%$ RSD \\
\hline Glucose & 36.9 & 38.2 & 37.9 & 37.7 & 0.7 & 1.8 \\
\hline Fructose & 41.6 & 38.9 & 39.1 & 39.9 & 1.5 & 3.7 \\
\hline
\end{tabular}

Concentration of monosaccharides based on peak area $(\mathrm{mg} / \mathrm{mL})$

\begin{tabular}{l|l|l|l|l|l|l}
\hline Glucose & 37.9 & 37.1 & 36.3 & 37.1 & 0.8 & 2.1 \\
\hline Fructose & 41.5 & 37.9 & 39.5 & 39.6 & 1.8 & 4.6 \\
\hline
\end{tabular}

\section{RID/HPLC SYSTEM}

Concentration of monosaccharides based on peak area $(\mathrm{mg} / \mathrm{mL})$

\begin{tabular}{l|c|c|c|c|c|c}
\hline & 1 & 2 & 3 & Average & SD & \% RSD \\
\hline Glucose & 39.6 & 40.0 & 40.2 & 39.9 & 0.7 & 0.8 \\
\hline Fructose & 39.5 & 39.9 & 38.2 & 39.2 & 0.9 & 2.3 \\
\hline
\end{tabular}

Concentration of monosaccharides based on peak area $(\mathrm{mg} / \mathrm{mL})$

\begin{tabular}{l|l|l|l|l|l|l}
\hline Glucose & 39.2 & 39.5 & 40.2 & 39.6 & 0.5 & 1.4 \\
\hline Fructose & 39.3 & 38.7 & 38.4 & 38.8 & 0.4 & 1.1 \\
\hline
\end{tabular}
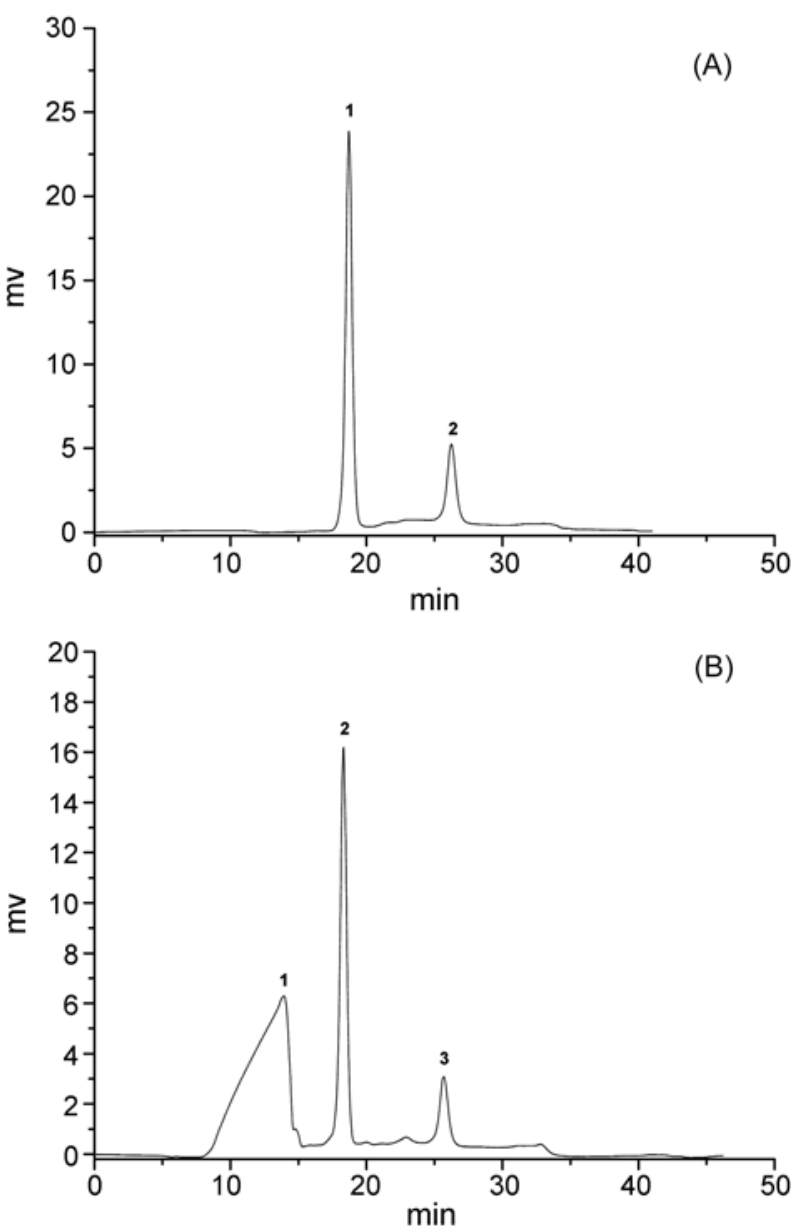

Figure 8. (A) The RID chromatogram of a standard. (B) The RID chromatogram of the hydrolyzed orange juice. The eluent was $100 \%$ water with the Supelcogel Pb column (7.8 mm I.D. $\times 300$ $\mathrm{mm}$ ) and the flow rate was $0.5 \mathrm{~mL} / \mathrm{min}$ at $85^{\circ} \mathrm{C}$. (A) 1 ; glucose 2 ; fructose (B) $1 ; \mathrm{Na}_{2} \mathrm{SO}_{4}$ (residual) 2; glucose 3; fructose.

concentrations were in good agreement between the two approaches.

\section{Conclusion}

We have applied ABEE derivatization for determination of fructose in hydrolysates of the yogurt and orange juice samples for the first time, and have successfully determined the concentrations of all monosaccharides including fructose. ABEE-fructose was detected as a doublet by UVDHPLC, and the peak ratio of the doublet was kept constant over a wide range of concentration. The validity of this approach was confirmed by comparing the above results with the results obtained by RID-HPLC for the same samples and by observing quite close coincidence between the two results.

Acknowledgment. This study was supported by the Korean Science and Engineering Foundation (R01-2003000-10030-0). The salary of Huazi Huang was partially supported by the Postdoctral Supporting Program of Inha University. 


\section{References}

1. Peris, M. In Handbook of Food Analysis; Nollet, L. M. L., Ed.; Marcel Dekker: New York, 1996; Vol 1, p 533.

2. Herbreteau, B. Analysis 1992, 20, 335.

3. Honda, S.; Akao, E.; Susuki, S.; Okuda, M.; Kakehi, K.; Nakumara, J. Anal. Chem. 1989, 180, 351.

4. Honda, S.; Susuki, S.; Nose, A.; Yamamoto, K.; Kakehi, K. Carbohydr. Res. 1991, 215, 193.

5. Guttman, A. J. Chromatogr. A 1997, 763, 271.

6. Koimur, M.; Lu, B.; Westerlund, D. Chromatographia 1996, 43, 254.

7. Rakotomanga, S.; Baillet, A.; Pellerin, F.; Baylocq-Ferrier, D. $J$.
Pharm. Biomed. Anal. 1992, 10, 587.

8. Zhang, R. E.; Cao, Y. L.; Hearn, M. W. Anal. Biochem. 1991, 195 , 160 .

9. Anumula, K. R. Anal. Biochem. 1994, 220, 275.

10. Kwon, H.; Kim, J. J. Liquid Chromatogr. 1995, 7, 1437.

11. Akiyama, T. J. Chromatogr. 1991, 588, 53.

12. Verzele, M.; Simones, G.; Van Dammed, F. Chromatographia 1987, 23, 291.

13. Kwon, H.; Kim, J. Anal. Biochem. 1993, 215, 243.

14. Wu, W.; Hamase, K.; Kiruchi, M.; Yakamoto, K.; Zaitsu, K. Anal. Sci. 2000, 16, 919 .

15. Ko, J. H.; Cheong, W. J. Bull. Korean Chem. Soc. 2001, 22, 123. 